\title{
ERBB2 in anti-EGFR-resistant colorectal cancer: cancer stem cells come into play
}

\author{
Sophie Mouillet-Richard
}

With over 900000 deaths per year worldwide in 2020 according to Globocan, colorectal cancer (CRC) represents the third deadliest cancer. Over the past 20 years, the median overall survival for CRC has substantially increased, especially in patients with metastatic disease, which has doubled to reach 30 months, as a result of the advent of targeted therapies. Despite this progress, the 5-year survival rate for patients with metastatic CRC remains below 15\%, calling for an improved management of these patients, which account for roughly $20 \%-30 \%$ of new diagnosed cases.

A consensus view is that CRC arises from uncontrolled expansion of intestinal stem cells (ISCs). ${ }^{1}$ By analogy with ISCs, CRC stem cells (CSCs) are endowed with self-renewal potential and display significant plasticity. They are believed to fuel CRC growth as well as metastasis, and display resistance to therapy, making their targeting one of the most critical challenges in the field of oncology. ${ }^{1}$ The study by Mangiapane et $a l^{2}$ tackles this issue by leveraging a collection of colorectal CSCs growing as spheroids and expressing the CSC marker CD44v6, which they previously showed to define a population of CSCs with enhanced metastatic potential. ${ }^{3}$ Among those, some bear RAS or BRAF mutations and were thoroughly resistant to the anti-epidermal growth factor receptor (EGFR) antibody cetuximab, as anticipated. Unexpectedly, a significant proportion of RAS and BRAF wild-type CSCs also displayed resistance to cetuximab. To shed some light on the mechanisms involved in this resistance phenomenon, they analysed differentially expressed genes between cetuximab-resistant and cetuximabsensitive CSCs, and found an increased expression of ERBB2, encoding HER2, a member of the EGFR family, in the resistant population. Building on their previous identification of the phosphatidylinositol 3-kinase (PI3K)/AKT pathway as an important regulator of CD44v6-positive

INSERM-U1138, Centre de Recherche des Cordeliers, Sorbonne Université, Université de Paris, Paris, France

Correspondence to Dr Sophie Mouillet-Richard, INSERM-U1138, Centre de Recherche des Cordeliers, Sorbonne Université, Université de Paris, Paris 75006, France; sophie.mouillet-richard@parisdescartes.fr cells survival, they went on to show that $E R B B 2$ is transcriptionally regulated by PI3K. In agreement with their previous study, ${ }^{3}$ PI3K inhibition efficiently eliminated disseminated cancer cells after injection in the spleen, while it has low efficacy when cells are injected subcutaneously. ${ }^{4}$ To account for this observation, they made the assumption that, within the tumorous tissue, additional signals emanating from the microenvironment may foster ERBB2 upregulation, and thereby protect cells from death induced by PI3K blockade. The authors focused on hepatocyte growth factor (HGF), osteopontin (OPN) and stromal-cell derived factor 1 (SDF-1), three soluble factors secreted by cancerassociated fibroblasts (CAFs), which they previously incriminated in the regulation of CD44v6 expression and in the promotion of an aggressive phenotype of CSCs on xenografting. ${ }^{3}$ Indeed, they found that these cytokines synergise to induce ERBB2 expression, making it insensitive to PI3K inhibition. This novel result actually adds another facet to the role described for these tumour microenvironment-derived molecules in supporting CSCs in CRC. The question then arose as to how design the appropriate combo to efficiently target these colorectal CSCs. Because these CAFs-derived cytokines activate the MAP kinase pathway, the authors considered the MEK kinase as potential candidate. In line with monotherapies being less efficient than drug combinations, the concomitant targeting of ERBB2 with trastuzumab, PI3K with either BKM120 or taselisib and MEK with trametinib or cobimetinib significantly hampered the tumour growth of CSCs cells injected subcutaneously. At the same time, this triplet of agents promoted a drastic reduction in CD44v6 staining in xenografts, suggesting a powerful anti-CSC activity.

Altogether, these enticing data consolidate the notion that activation of HER2 signalling contributes to cetuximab resistance in CRC, and that this may arise as a result of increased ERBB2 expression, beyond ERBB2 amplification $^{56}$ or mutations, ${ }^{7}$ as also recently demonstrated by Lupo et al. ${ }^{8}$ These results and the Lupo et al study ${ }^{8}$ thus advocate for a comprehensive analysis of ERBB2, including mRNA expression, in EGFR-agents refractory settings, and may set the stage for a wider use of anti-ERBB2-based therapies to treat metastatic CRC. Interestingly, the study by Lupo et al documents that ERBB2 is upregulated in colorectal residual disease and that the corresponding drug tolerant cells display hallmarks of DNA labelretaining cells, themselves being secretory precursors committed to mature into Paneth cells. ${ }^{8}$ The question then arises as to whether the ERBB2-positive cell population described by Lupo and colleagues ${ }^{8}$ shares some common features with the ERBB2-expressing CSCs of the Mangiapane et al study. ${ }^{2}$ Such a hypothesis would notably fit with the well-established reciprocal relationship between CSCs and drug resistance. Another noteworthy point of convergence in the two studies relates to the signalling pathways involved: both incriminate MEK and PI3K, which may operate both upstream and downstream from ERBB2 and thereby maintain a positive feedback loop. Thus, the study by Lupo et $a l^{8}$ directly supports the triplet strategy-combined targeting of ERBB2, MEK and PI3K- proposed by Mangiapane and colleagues. ${ }^{2}$ Of note, the synergistic use of PI3K and pan-ERBB inhibitors has previously been reported to be efficient in the context of mutant KRAS CRC. ${ }^{9}$ However, in contrast to, ${ }^{2}$ the rationale for the dual targeting of PI $3 \mathrm{~K}$ and ERBB in the latter study was based on the increased expression and activity of ERBB family members in mutant KRAS CRC resistant to $\mathrm{PI} 3 \mathrm{~K} / \mathrm{mTOR}$ inhibition, as a result of FOXO3 activation. ${ }^{9}$ Nevertheless, in line with, ${ }^{2} \mathrm{PI} 3 \mathrm{~K} / \mathrm{mTOR}$ resistant, ERBBhyper-activated CRC cells were found to exhibit stem-cell like characteristics. ${ }^{9}$

However, attractive the combined ERBB2, MEK and PI3K therapeutic strategy may appear, some open questions remain. Should not the triplet strategy be compared with the dual targeting of MEK and PI3K, since the latter appears to be more efficient according to cell-based xenograft assays? ${ }^{10}$ How can we reconcile the observation that CRC cells engineered to overexpress ERBB2 lose markers of epithelial to mesenchymal transition (EMT) as well as invasive and migratory properties ${ }^{10}$ with the report that CD44v6positive cells, which express abundant levels of $\mathrm{ERBB}^{2}$ display hallmarks of EMT and are endowed with metastatic properties? ${ }^{3}$ At a mechanistic level, it is unclear what other ERBB receptor ERBB2 dimerises with in CD44v6-positive cells. Answer to this question may help further refine therapeutic schemes.

In summary, notwithstanding these issues, the study by Mangiapane et $a l^{2}$ 
brings to light an unprecedented link between resistance to cetuximab and the upregulation of ERBB2 in the CSC compartment of CRC. Most importantly, it clearly offers new therapeutic prospects for the management of patients who have become refractory to EGFR-targeted agents. In addition, it may give a second wind to ERBB2-based clinical trials such as the HERACLES study. ${ }^{11}$ The combination of trastuzumab to MEK and PI3K inhibitors advocated by Mangiapane and colleagues ${ }^{2}$ may notably provide an adequate response to the development of resistance to ERBB2 blockade, potentially involving these effectors as recently suggested. $^{11}$

Contributors SM-R wrote the article.

Funding The author has not declared a specific grant for this research from any funding agency in the public, commercial or not-for-profit sectors.

Competing interests None declared.

Patient consent for publication Not required.

Provenance and peer review Commissioned; externally peer reviewed.

(c) Author(s) (or their employer(s)) 2021. No commercial re-use. See rights and permissions. Published by BMJ.

\section{Check for updates}

To cite Mouillet-Richard S. Gut Epub ahead of print: [please include Day Month Year]. doi:10.1136/ gutjnl-2020-323924

Received 19 January 2021

Revised 10 February 2021

Accepted 11 February 2021

\section{C) Linked}

- http://dx.doi.org/10.1136/gutjnl-2020-323553

Gut 2021;0:1-2.

doi:10.1136/gutjnl-2020-323924

ORCID iD

Sophie Mouillet-Richard http://orcid.org/0000-00028950-1949

\section{REFERENCES}

1 van der Heijden M, Vermeulen L. Stem cells in homeostasis and cancer of the gut. Mol Cancer 2019;18:66.

2 Mangiapane LR, Nicotra A, Turdo A. PI3K-driven HER2 expression is a potential therapeutic target in colorectal cancer stem cells. Gut. doi:10.1136/ gutjnl-2020-323553

3 Todaro M, Gaggianesi M, Catalano V, et al. Cd44V6 is a marker of constitutive and reprogrammed cancer stem cells driving colon cancer metastasis. Cell Stem Cell 2014;14:342-56.
4 Veschi V, Mangiapane LR, Nicotra A, et al. Targeting chemoresistant colorectal cancer via systemic administration of a BMP7 variant. Oncogene 2020;39:987-1003.

5 Bertotti A, Migliardi G, Galimi F, et al. A molecularly annotated platform of patient-derived xenografts ("xenopatients") identifies HER2 as an effective therapeutic target in cetuximab-resistant colorectal cancer. Cancer Discov 2011;1:508-23.

6 Yonesaka K, Zejnullahu K, Okamoto I, et al. Activation of ErbB2 signaling causes resistance to the EGFRdirected therapeutic antibody cetuximab. Sci Trans/ Med 2011;3:99ra86.

7 Bertotti A, Papp E, Jones S, et al. The genomic landscape of response to EGFR blockade in colorectal cancer. Nature 2015;526:263-7.

8 Lupo B, Sassi F, Pinnelli M, et al. Colorectal cancer residual disease at maximal response to EGFR blockade displays a druggable Paneth cell-like phenotype. Sci Trans/ Med 2020;12. doi:10.1126/ scitranslmed.aax8313. [Epub ahead of print: 05 Aug 2020].

9 Belmont PJ, Jiang P, McKee TD, et al. Resistance to dual blockade of the kinases PI3K and mTOR in KRASmutant colorectal cancer models results in combined sensitivity to inhibition of the receptor tyrosine kinase EGFR. Sci Signal 2014;7:ra107.

10 Belli V, Matrone N, Napolitano S, et al. Combined blockade of MEK and PI3KCA as an effective antitumor strategy in HER2 gene amplified human colorectal cancer models. J Exp Clin Cancer Res 2019;38:236.

11 Siena S, Sartore-Bianchi A, Marsoni S, et al. Targeting the human epidermal growth factor receptor 2 (HER2) oncogene in colorectal cancer. Annals of Oncology 2018;29:1108-19. 